\title{
Article \\ Cayley Graphs Defined by Systems of Equations
}

\author{
Fuyuan Yang ${ }^{1}$, Qiang Sun ${ }^{2}\left(\mathbb{D}\right.$, Hongbo Zhou $^{3}$ and Chao Zhang ${ }^{1, *}$ (D) \\ 1 School of Mathematics and Statistics, Guizhou University, Guiyang 550025, China; fyyang9@163.com \\ 2 School of Mathematical Science, Yangzhou University, Yangzhou 225002, China; qsun1987@163.com \\ 3 College of Mathematics and Information Science, Guiyang University, Guiyang 550005, China; \\ zhb5278@163.com \\ * Correspondence: zhangc@amss.ac.cn
}

check for updates

Citation: Yang, F.; Sun, Q.; Zhou, H.; Zhang, C. Cayley Graphs Defined by Systems of Equations. Axioms 2022, 11, 100. https://doi.org/10.3390/ axioms 11030100

Academic Editors: Takao Komatsu, Claudio Pita-Ruiz and Ram Krishna Pandey

Received: 19 December 2021

Accepted: 22 February 2022

Published: 25 February 2022

Publisher's Note: MDPI stays neutral with regard to jurisdictional claims in published maps and institutional affiliations.

Copyright: () 2022 by the authors Licensee MDPI, Basel, Switzerland. This article is an open access article distributed under the terms and conditions of the Creative Commons Attribution (CC BY) license (https:// creativecommons.org/licenses/by/ $4.0 /)$.

\begin{abstract}
Let $R$ be a finite ring. In this paper, we mainly explore the conditions to ensure the graph $B \Gamma_{n}$ defined by a system of equations $\left\{f_{i} \mid i=2, \ldots, n\right\}$ to be a Cayley graph or a Hamiltonian graph. More precisely, we prove that $B \Gamma_{n}$ is a Cayley graph with $G=\langle\phi, A\rangle$ a group of dihedral type if and only if the system $\mathcal{F}_{n}=\left\{f_{i} \mid i=2, \ldots, n\right\}$ is Cayley graphic of dihedral type in $R$. As an application, the well-known Lovász Conjecture, which states that any finite connected Cayley graph has a Hamilton cycle, holds for the connected $B \Gamma_{n}$ defined by Cayley graphic system $\mathcal{F}_{n}$ of dihedral type in the field $G F\left(p^{k}\right)$.
\end{abstract}

Keywords: Cayley graph; hamiltonian graph; dihedral type; Hamilton-laceable

MSC: 05C25; 05C45; 17B67

\section{Introduction}

The graph $B \Gamma_{n}\left(R ; f_{2}, \ldots, f_{n}\right)$ defined by a system of equations over a finite ring $R$ have been extensively studied and used since the 1990s. The construction of graph $B \Gamma_{n}\left(R ; f_{2}, \ldots, f_{n}\right)$ is motivated by the problems from extremal graph theory (see [1,2] for details), and the concrete construction was originally due to [3]. The construction of the graphs by systems of equations provides a useful tool to study graph theory, for instance, constructing the graphs $D(k, q)$ and $C D(k, q)$, to approximate the structure and behavior of incidence graphs of regular generalized polygons [4], establishing the lower bound of the Ramsey numbers of graphs associated to generalized Kac-Moody algebras of rank two [5] and hypergraphs [6], and constructing certain graphs with given properties [1,7,8]. In addition, Lazebnik and Woldar [3] established some important properties including regularity and bi-regularity, existence of special vertex colorings, and existence of covering maps of $B \Gamma_{n}\left(R ; f_{2}, \ldots, f_{n}\right)$. Additional properties of $B \Gamma_{n}\left(R ; f_{2}, \ldots, f_{n}\right)$ can be found in the survey [9] and the references therein. Moreover, the girth $[8,10-12]$ and the classification up to isomorphisms [13] of graph $B \Gamma_{n}\left(R ; f_{2}, \ldots, f_{n}\right)$ are further considered recently.

It is natural to study other properties of graph $B \Gamma_{n}\left(R ; f_{2}, \ldots, f_{n}\right)$. For example, Lazebnik et al. [14] explored the condition to ensure the graphs to be semisymmetric or connected. They proved that $B \Gamma_{n}\left(R ; p_{1} l_{1}, \ldots, p_{1} l_{i}, \ldots, p_{1} l_{n-1}\right)$ is semisymmetric if $R=G F\left(p^{k}\right)$ is a finite field for any $n \geq 3$ and $p \geq 3$. Moreover, some open problems of $B \Gamma_{n}\left(R ; f_{2}, \ldots, f_{n}\right)$ were raised up in [3] including when $B \Gamma_{n}\left(R ; f_{2}, \ldots, f_{n}\right)$ is a Cayley graph or Hamiltonian graph. Cayley graphs are those graphs whose vertices are identified with elements of groups and adjacency relations are defined by subsets of the groups.

The present paper aims to study the question of when $B \Gamma_{n}\left(R ; f_{2}, \ldots, f_{n}\right)$ is a Cayley graph. To be more precise, we consider a class of function systems and we call them Cayley graphic function systems of dihedral type, which ensures $B \Gamma_{n}$ to be a Cayley graph. The main theorem is as follows. 
Theorem 1. The graph $B \Gamma_{n}$ is a Cayley graph on some group $G=\langle\phi, A\rangle$ of dihedral type with $\phi:(p) \leftrightarrow[p] \in \operatorname{Aut}\left(B \Gamma_{n}\right)$ and $A$ uniform if and only if the function system $\mathcal{F}_{n}=\left\{f_{i} \mid i=\right.$ $2, \ldots, n\}$ is Cayley graphic of dihedral type (see Definition 5) in $R$.

Cayley graphs are widely studied together with Hamiltonian graphs (see $[15,16]$ for example), where Hamiltonian graphs are those graphs having a cycle visiting every vertex exactly once. A variant of the well-known Lovász Conjecture on Hamiltonian paths states that every finite connected Cayley graph has a Hamilton cycle. Many people focus on discussing the special case of the above conjecture (see [15,17-20]). We pay attention to these results and then study the Hamiltonicity of graph $B \Gamma_{n}\left(R ; f_{2}, \ldots, f_{n}\right)$.

Furthermore, we consider the Hamiltonicity of this class of graphs and we have two following theorems; note that Theorem 3 supports the Lovász Conjecture.

Theorem 2. Let $R$ be a finite ring with $|R|$ an even positive integer, and $B \Gamma_{n}$ be a connected graph detemined by the function system $\mathcal{F}_{n}=\left\{f_{i} \mid i=2, \ldots, n\right\}$, which is Cayley graphic of dihedral type. Then $B \Gamma_{n}$ is Hamilton-laceable, i.e., for any vertices $u$ and $v$ from two distinct parts in the bipartite graph $B \Gamma_{n}$, there is a Hamilton path whose terminal vertices are $u$ and $v$.

Theorem 3. Let $R=G F\left(p^{k}\right)$ be a field, for some prime $p$. If $\mathcal{F}_{n}=\left\{f_{i} \mid i=2, \ldots, n\right\}$ is Cayley graphic of dihedral type in $R$ and $B \Gamma_{n}$ connected, then $B \Gamma_{n}$ has a Hamilton cycle.

The paper is organized as follows: in Section 2, we recall the definition and some properties of the graph $B \Gamma_{n}\left(R ; f_{2}, \ldots, f_{n}\right)$. We prove our main theorem in Section 3, i.e., the function system $\left\{f_{i} \mid i=2, \ldots, n\right\}$ is Cayley graphic of dihedral type in $R$ if and only if $B \Gamma_{n}$ is a Cayley graph with the group $G=\langle\phi, A\rangle$ is of dihedral type with $A$ satisfying so-called uniform condition (see Definition 4 ). The last section of this paper mainly study the Hamiltonicity of graph $B \Gamma_{n}\left(R ; f_{2}, \ldots, f_{n}\right)$.

\section{The Graphs Defined by Systems of Equations}

In this section, we will recall the definition of the graph defined by systems of equations.

Throughout this paper, for a graph $\Gamma$, we always denote by $V(\Gamma)$ the vertex set and by $E(\Gamma)$ the edge set. The element of $E(\Gamma)$ connecting two vertices $u, v \in V(\Gamma)$ will be written as $u \sim v$.

Definition 1. ([3], Section 2.1) Let $f_{i}: R^{2 i-2} \rightarrow R$ be an arbitrary function, $2 \leq i \leq n$. We define the bipartite graph $B \Gamma_{n}=B \Gamma_{n}\left(R ; f_{2}, \ldots, f_{n}\right)$ as follows. The set of vertices $V\left(B \Gamma_{n}\right)$ is the disjoint union of two copies of $R^{n}$, one denoted by $P_{n}$ and the other by $L_{n}$. Elements of $P_{n}$ will be called points and those of $L_{n}$ lines. In order to distinguish points from lines we introduce the use of parentheses and brackets: if $a \in R^{n}$, then $(a) \in P_{n}$ and $[a] \in L_{n}$. We define edges of $B \Gamma_{n}$ by declaring point $(p)=\left(p_{1}, p_{2}, \ldots, p_{n}\right)$ and line $[l]=\left[l_{1}, l_{2}, \ldots, l_{n}\right]$ to be adjacent if and only if the following $n-1$ relations on their coordinates hold:

$$
\begin{aligned}
p_{2}+l_{2}= & f_{2}\left(p_{1}, l_{1}\right), \\
p_{3}+l_{3}= & f_{3}\left(p_{1}, l_{1}, p_{2}, l_{2}\right), \\
\ldots & \ldots \\
p_{n}+l_{n}= & f_{n}\left(p_{1}, l_{1}, p_{2}, l_{2}, \ldots, p_{n-1}, l_{n-1}\right) .
\end{aligned}
$$

For a function $f_{i}: R^{2 i-2} \rightarrow R$, we define $\bar{f}_{i}: R^{2 i-2} \rightarrow R$ by the rule $\bar{f}_{i}\left(x_{1}, y_{1}, \ldots, x_{i-1}, y_{i-1}\right)$ $=f_{i}\left(y_{1}, x_{1}, \ldots, y_{i-1}, x_{i-1}\right)$. We call $f_{i}$ symmetric if the functions $f_{i}$ and $\bar{f}_{i}$ coincide.

Evidently, if the order of $R$ is $|R|=r$, then the number of vertices of graph $B \Gamma_{n}$ is $2 r^{n}$, i.e., $\left|V\left(B \Gamma_{n}\right)\right|=2 r^{n}$.

There is another fundamental family of graphs as follows if all functions are symmetric. 
Definition 2. ([3], Section 2.2) Let $f_{i}: R^{2 i-2} \rightarrow R$ be symmetric for all $2 \leq i \leq n$. We define $\Gamma_{n}=\Gamma\left(R ; f_{2}, \ldots, f_{n}\right)$ to be the graph with vertex set $V\left(\Gamma_{n}\right)=R^{n}$, where distinct vertices (vectors) $a=\left\langle a_{1}, a_{2}, \ldots, a_{n}\right\rangle$ and $b=\left\langle b_{1}, b_{2}, \ldots, b_{n}\right\rangle$ are adjacent if and only if the following $n-1$ relations on their coordinates hold:

$$
\begin{aligned}
a_{2}+b_{2}= & f_{2}\left(a_{1}, b_{1}\right), \\
a_{3}+b_{3}= & f_{3}\left(a_{1}, b_{1}, a_{2}, b_{2}\right), \\
\ldots & \ldots \\
a_{n}+b_{n}= & f_{n}\left(a_{1}, b_{1}, a_{2}, b_{2}, \ldots, a_{n-1}, b_{n-1}\right) .
\end{aligned}
$$

Let $\Gamma=(V, E)$ be a graph. We say that $\Gamma$ has a loop at vertex $v$ provided that there is an edge $e \in E$ connecting $v$ and itself. The following proposition can be deduced directly by the definition, see [3,9] for details and more properties of $B \Gamma_{n}$ and $\Gamma_{n}$.

Proposition 1. ([3], Proposition 2) If char $R=2, \Gamma_{n}$ has a loop at vertex $\langle a\rangle$ if and only if

$$
f_{i}\left(a_{1}, a_{1}, \ldots, a_{i-1}, a_{i-1}\right)=0
$$

for all $2 \leq i \leq n$.

Now we recall the definitions related to the automorphism group.

Definition 3. ([21], Section 15) An automorphism of a simple graph $\Gamma$ is a bijection $\sigma$ : $V(\Gamma) \rightarrow V(\Gamma)$ such that vertex $u$ is adjacent to $v$ is an edge of $\Gamma$ if and only if $\sigma(u)$ is adjacent to $\sigma(v)$ is an edge of $\Gamma$.

The group consisting of all automorphisms of $\Gamma$ is called the automorphism group of $\Gamma$, and we denote it by $\operatorname{Aut}(\Gamma)$.

The following example appears in Section 3.5 of paper [9].

Example 1. For any $x \in R$, the map $\sigma_{n, x}$ is an automorphism of $B \Gamma_{n}$, where

$$
\begin{gathered}
\sigma_{n, x}((p))=\left(p_{1}, \ldots, p_{n-1}, p_{n}+x\right), \\
\sigma_{n, x}([l])=\left[l_{1}, \ldots, l_{n-1}, l_{n}-x\right],
\end{gathered}
$$

for any $(p) \in P_{n,}[l] \in L_{n}$

For convenience, we denote by $(p)_{k}$ (resp. $\left.[l]_{k}\right)$ the $k$-th coordinate of $(p)$ (resp. [l]), and by $(p(i), l(i))$ the sequence of variables $\left(p_{1}, l_{1}, \ldots, p_{i-1}, l_{i-1}\right)$ for any $i=2,3, \ldots, n$, where $(p)=\left(p_{1}, . ., p_{n}\right)$ and $[l]=\left[l_{1}, \ldots, l_{n}\right]$.

Definition 4. An automorphism $\sigma \in \operatorname{Aut}\left(B \Gamma_{n}\right)$ is uniform if

(1) $\sigma$ maps points to points and lines to lines, i.e., $\sigma((p))=\left(p^{\prime}\right), \sigma([l])=\left[l^{\prime}\right]$;

(2) $\sigma((p))_{k}=p_{k}+x$ and $\sigma((p))_{j}=p_{j}$ for all $(p) \in P_{n}$ if there is some $k \in\{1,2, \cdots, n\}$ such that $\sigma((0))_{j}=0$ for all $1 \leq j<k$, and $\sigma((0))_{k}=x \in R$;

(3) for all $(p),[l]$ and $k<i \leq n$, we have

$$
f_{i}\left(p^{\prime}(i), l^{\prime}(i)\right)-\left(p^{\prime}\right)_{i}-\left[l^{\prime}\right]_{i}=f_{i}(p(i), l(i))-(p)_{i}-[l]_{i},
$$

where $\left(p^{\prime}\right)=\sigma((p)),\left[l^{\prime}\right]=\sigma([l])$.

Moreover, a subset $S \subseteq \operatorname{Aut}\left(B \Gamma_{n}\right)$ is called to be uniform if $S$ consists of uniform automorphisms. 
Remark 1. In the above definition,

(1) for the second condition, $k$ and $x$ are not necessary to be unique and we can take the identity automorphism for example;

(2) the third condition can be seem as a generalization of automorphism of graph $B \Gamma_{n}$, as any automorphism $\sigma$ of graph $B \Gamma_{n}$ satisfies that both the left side and the right side of Equation (1) equal to 0 for two adjacent points $(p)$ and $[l]$.

Now we introduce Cayley graphic functions of dihedral type.

Definition 5. We call the function system $\mathcal{F}_{n}=\left\{f_{i} \mid i=2, \ldots, n\right\}$ to be Cayley graphic of dihedral type in $R$, if there is a uniform subset $X=\left\{\tau_{k, x} \mid x \in R, k=1, \ldots, n\right\} \subseteq \operatorname{Aut}\left(B \Gamma_{n}\right)$ and $\phi:(p) \leftrightarrow[p]$ lies in $\operatorname{Aut}\left(B \Gamma_{n}\right)$, such that

(1) $\tau_{k, x}((p))_{k}=p_{k}+x$ and $\tau_{k, x}([l])_{k}=l_{k}-x$;

(2) $\tau_{k, x}((p))_{j}=p_{j}$ and $\tau_{k, x}([l])_{j}=l_{j}$ for all $1 \leq j<k$;

(3) $\tau_{k,-x}([p])_{j}=\tau_{k, x} \phi([p])_{j}$ for all $k+1 \leq j \leq n$.

We have a property for Cayley graphic functions of dihedral type in $R$.

Proposition 2. Let the function system $\mathcal{F}_{n}=\left\{f_{i} \mid i=2, \ldots, n\right\}$ be Cayley graphic of dihedral type in $R$. Then there is a uniform subset $X^{\prime}=\left\{\sigma_{k, x} \mid x \in R, k=1, \ldots, n\right\} \subseteq \operatorname{Aut}\left(B \Gamma_{n}\right)$, such that

(1) $\quad \sigma_{k, x}((p))_{j}=\tau_{k, x}((p))_{j}$ and $\sigma_{k, x}([l])_{j}=\tau_{k, x}([l])_{j}$ for all $j \leq k$;

(2) $\quad \sigma_{k,-x}([p])_{j}=\sigma_{k, x}((p))_{j}$ for all $k+1 \leq j \leq n$;

(3) when $j>k$, the sum of all odd functions $h(x)$ (i.e. $h(x)=-h(-x)$ ) in the additive terms of $\sigma_{k, x}((p))_{j}$ is 0 .

Proof of Proposition 2. Evidently, $\tau_{k, x} \tau_{k^{\prime}, z}$ is uniform if $\tau_{k, x}$ and $\tau_{k^{\prime}, z}$ are uniform (by a straightforward check). When $j>k$, if the sum of all odd functions in the additive terms of $\sigma_{k, x}((p))_{j}$ is $h(x)$, which is nonzero, we replace the automorphism $\tau_{k, x}$ with $\sigma_{k, x}=\tau_{j,-h(x)} \tau_{k, x}$. Repeating the replacement finitely many steps, we obtain the automorphisms as required.

In what follows, for Cayley graphic function system of dihedral type, we always take uniform automorphisms $X^{\prime}=\left\{\sigma_{k, x} \mid x \in R, k=1, \ldots, n\right\} \subseteq \operatorname{Aut}\left(B \Gamma_{n}\right)$ in the previous proposition.

We further explain the definition and introduce the functions $b_{i, k}$ and $y_{i, k}$, associated to $\left\{f_{i} \mid i=2, \ldots, n\right\}$.

Remark 2. By Proposition 1 in [3], if $\left\{f_{i} \mid i=2, \ldots, n\right\}$ are symmetric then $\phi:(p) \leftrightarrow[p]$ is an automorphism. Moreover, $(p) \sim[l]$ if and only if $\sigma_{k, x}((p)) \sim \sigma_{k, x}([l])$ for $\sigma_{k, x} \in X^{\prime}$. Now we set

$$
\begin{aligned}
b_{i, k}\left(p_{1}, \ldots, p_{i-1}, x\right) & :=\sigma_{k, x}((p))_{i}-p_{i}, \\
y_{i, k}\left(l_{1}, \ldots, l_{i-1}, x\right) & :=\sigma_{k, x}([l])_{i}-l_{i},
\end{aligned}
$$

for all $i=k+1, \ldots, n$. Let $\left(p^{\prime}\right)_{m}=\sigma_{k, x}((p))_{m}$ and $\left[l^{\prime}\right]_{m}=\sigma_{k, x}([l])_{m}$ for all $1 \leq m \leq n$. Since $X^{\prime}=\left\{\sigma_{k, x} \mid x \in R, k=1, \ldots, n\right\}$ is uniform, by Definition 4 (3) follows

$$
b_{i, k}\left(p_{1}, \ldots, p_{i-1}, x\right)+y_{i, k}\left(l_{1}, \ldots, l_{i-1}, x\right)=f_{i}\left(p^{\prime}(i), l^{\prime}(i)\right)-f_{i}(p(i), l(i))
$$

for all $(p) \in P_{n}$ and $[l] \in L_{n}$.

For convenience, we write $b_{i, k}$ and $y_{i, k}$ for short unless stated otherwise. Note that the existence of functions $b_{i, k}$ and $y_{i, k}$ is equivalent to the existence of $\sigma_{k, x}$. 
Example 2. If $n=2, f_{2}\left(p_{1}, l_{1}\right)=p_{1}+l_{1}$, then

$$
b_{2,1}+y_{2,1}=f_{2}\left(p_{1}+x, l_{1}-x\right)-f_{2}\left(p_{1}, l_{1}\right)=0 .
$$

Set $b_{2,1}=y_{2,1}=0$. It is straightforward to check that $f_{2}\left(p_{1}, l_{1}\right)=p_{1}+l_{1}$ is Cayley graphic of dihedral type in $R$.

By Definition 5, Proposition 2, and Remark 2, we obtain the uniqueness of $b_{i, k}$ and $y_{i, k}$ for Cayley graphic functions of dihedral type in $R$.

Proposition 3. If $\mathcal{F}_{n}=\left\{f_{i} \mid i=2, \ldots, n\right\}$ is Cayley graphic of dihedral type in $R$, then (in terms of the above notations)

(1) the functions $b_{i, k}$ and $y_{i, k}$ are unique;

(2) we have $b_{i, k}\left(p_{1}, \ldots, p_{i-1}, 0\right)=y_{i, k}\left(l_{1}, \ldots, l_{i-1}, 0\right)=0$;

(3) for the case $n=2$, the functions $b_{2,1}$ and $y_{2,1}$ are additive, i.e., if $b_{2,1}^{\prime}$ and $y_{2,1}^{\prime}$ are functions associated to $f_{2}^{\prime}$ in another function system $\mathcal{F}_{2}^{\prime}$ that is Cayley graphic of dihedral type, then $b_{2,1}^{\prime}+b_{2,1}$ and $y_{2,1}^{\prime}+y_{2,1}$ are functions associated to $f_{2}^{\prime}+f_{2}$;

(4) the system $\mathcal{F}_{j}=\left\{f_{i} \mid i=2, \ldots, j\right\}$ is Cayley graphic of dihedral type for $j \leq n$.

Proof of Proposition 3. (1) Let us assume the opposite and let $b_{i, k}, b_{i, k}^{\prime}$ and $y_{i, k}, y_{i, k}^{\prime}$ be the functions associated to $\left\{f_{i} \mid i=2, \ldots, n\right\}$, respectively. Now we set

$$
\begin{aligned}
& \bar{b}_{i, k}=b_{i, k}^{\prime}-b_{i, k}, \\
& \bar{y}_{i, k}=y_{i, k}^{\prime}-y_{i, k} .
\end{aligned}
$$

By Remark 2,

$$
\begin{aligned}
& \bar{b}_{k+1, k}\left(p_{1}, \ldots, p_{k}, x\right)=\bar{y}_{k+1, k}\left(p_{1}, \ldots, p_{k},-x\right), \\
& \bar{b}_{k+1, k}\left(p_{1}, \ldots, p_{k}, x\right)+\bar{y}_{k+1, k}\left(l_{1}, \ldots, l_{k}, x\right)=0,
\end{aligned}
$$

for all $(p) \in P_{n}$ and $[l] \in L_{n}$. We have

$$
\bar{y}_{k+1, k}\left(p_{1}, \ldots, p_{k},-x\right)+\bar{y}_{k+1, k}\left(l_{1}, \ldots, l_{k}, x\right)=0 .
$$

Then there is an odd function $h(x)$ such that

$$
\begin{aligned}
& \bar{b}_{k+1, k}=b_{k+1, k}^{\prime}-b_{k+1, k}=h(x), \\
& \bar{y}_{k+1, k}=y_{k+1, k}^{\prime}-y_{k+1, k}=h(-x) .
\end{aligned}
$$

By Proposition 2 (3), we have

$$
\begin{aligned}
& b_{k+1, k}^{\prime}=b_{k+1, k} \\
& y_{k+1, k}^{\prime}=y_{k+1, k} .
\end{aligned}
$$

Now we prove the uniqueness of $b_{i, k}$ and $y_{i, k}$ by induction on $i$. Suppose that it holds for all $k+1 \leq j \leq i-1$, i.e., $b_{j, k}^{\prime}=b_{j, k}, y_{j, k}^{\prime}=y_{j, k}$, it suffice to prove it for the case $i$. Similarly, we have

$$
\begin{aligned}
& \bar{b}_{i, k}\left(p_{1}, \ldots, p_{i-1}, x\right)=\bar{y}_{i, k}\left(p_{1}, \ldots, p_{i-1},-x\right), \\
& b_{i, k}+y_{i, k}=b_{i, k}^{\prime}+y_{i, k}^{\prime}=f_{i}\left(p^{\prime}(i), l^{\prime}(i)\right)-f_{i}(p(i), l(i)),
\end{aligned}
$$

where $\left(p^{\prime}\right)=\sigma_{k, x}((p)),\left[l^{\prime}\right]=\sigma_{k, x}([l])$. Then

$$
\begin{aligned}
& \bar{b}_{i, k}\left(p_{1}, \ldots, p_{i-1}, x\right)=\bar{y}_{i, k}\left(p_{1}, \ldots, p_{i-1},-x\right) \\
& \bar{b}_{i, k}\left(p_{1}, \ldots, p_{i-1}, x\right)+\bar{y}_{i, k}\left(l_{1}, \ldots, l_{i-1}, x\right)=0
\end{aligned}
$$


Then, there is an odd function $h(x)$ such that

$$
\begin{aligned}
& \bar{b}_{i, k}=b_{i, k}^{\prime}-b_{i, k}=h(x), \\
& \bar{y}_{i, k}=y_{i, k}^{\prime}-y_{i, k}=h(-x) .
\end{aligned}
$$

By Proposition 2 (3), we have

$$
\begin{aligned}
& b_{i, k}^{\prime}=b_{i, k}, \\
& y_{i, k}^{\prime}=y_{i, k} .
\end{aligned}
$$

(2) Since

$$
\begin{aligned}
& b_{k+1, k}\left(p_{1}, \ldots, p_{k}, 0\right)=y_{k+1, k}\left(p_{1}, \ldots, p_{k}, 0\right), \\
& b_{k+1, k}\left(p_{1}, \ldots, p_{k}, 0\right)+y_{k+1, k}\left(l_{1}, \ldots, l_{k}, 0\right)=0,
\end{aligned}
$$

we have $2 b_{k+1, k}\left(p_{1}, \ldots, p_{k}, 0\right)=0$. If $b_{k+1, k}\left(p_{1}, \ldots, p_{k}, 0\right) \neq 0$, we let

$$
b_{k+1, k}^{\prime}\left(p_{1}, \ldots, p_{k}, x\right)=b_{k+1, k}\left(p_{1}, \ldots, p_{k}, x\right)+b_{k+1, k}\left(p_{1}, \ldots, p_{k}, 0\right),
$$

thus $b_{k+1, k}\left(p_{1}, \ldots, p_{k}, 0\right)=0$ by $(1)$.

We have

$$
\begin{aligned}
& b_{i, k}\left(p_{1}, \ldots, p_{i-1}, 0\right)=y_{k+1, k}\left(p_{1}, \ldots, p_{i-1}, 0\right), \\
& b_{i, k}\left(p_{1}, \ldots, p_{i-1}, 0\right)+y_{k+1, k}\left(l_{1}, \ldots, l_{i-1}, 0\right)=0,
\end{aligned}
$$

then $2 b_{i, k}\left(p_{1}, \ldots, p_{i-1}, 0\right)=0$. If $b_{i, k}\left(p_{1}, \ldots, p_{i-1}, 0\right) \neq 0$, we let

$$
b_{i, k}^{\prime}\left(p_{1}, \ldots, p_{i-1}, x\right)=b_{i, k}\left(p_{1}, \ldots, p_{i-1}, x\right)+b_{i, k}\left(p_{1}, \ldots, p_{i-1}, 0\right) .
$$

By induction on $i$ and (1), we have $b_{i, k}\left(p_{1}, \ldots, p_{i-1}, 0\right)=y_{i, k}\left(p_{1}, \ldots, p_{i-1}, 0\right)=0$.

(3) We have

$$
\begin{aligned}
& b_{2,1}+y_{2,1}=f_{2}\left(p_{1}+x, l_{1}-x\right)-f_{2}\left(p_{1}, l_{1}\right), \\
& b_{2,1}^{\prime}+y_{2,1}^{\prime}=f_{2}^{\prime}\left(p_{1}+x, l_{1}-x\right)-f_{2}^{\prime}\left(p_{1}, l_{1}\right) .
\end{aligned}
$$

Sum them up, and we have

$$
\left(b_{2,1}^{\prime}+b_{2,1}\right)+\left(y_{2,1}^{\prime}+y_{2,1}\right)=\left(f_{2}^{\prime}+f_{2}\right)\left(p_{1}+x, l_{1}-x\right)-\left(f_{2}^{\prime}+f_{2}\right)\left(p_{1}, l_{1}\right) .
$$

By (1), then $b_{2,1}^{\prime}+b_{2,1}$ and $y_{2,1}^{\prime}+y_{2,1}$ are functions associated to Cayley graphic of dihedral type $f_{2}^{\prime}+f_{2}$.

(4) The unique functions $b_{i, k}$ and $y_{i, k}$ are functions associated to Cayley graphic function system $\left\{f_{i} \mid i=2, \ldots, j\right\}$ of dihedral type, for $j \leq n$.

\section{A Condition for Graphs $B \Gamma_{n}$ to be Cayley Graphs}

In this section, we provide a condition to ensure a graph $B \Gamma_{n}$ to be a Cayley graph. We first recall the definition of Cayley graph.

Definition 6. ([16], Section 1) Let $G$ be a group and $S$ be a subset of $G$. A digraph $\Gamma=\operatorname{Cay}(G, S)$ is a Cayley graph with vertex set $G$ and two vertices $u, v \in G$ are adjacent if and only if $v u^{-1} \in S$. Moreover, if for any element $s \in S$, the inverse $s^{-1}$ also lies in $S$, then the adjacency is symmetric and thus the Cayley graph $\operatorname{Cay}(G, S)$ may be viewed as an undirected graph.

Obviously, the subset $S$ contains the identity of $G$ if and only if the Cayley graph Cay $(G, S)$ contains a loop at every vertex. 
Definition 7. Let $\Gamma$ be a graph and $H$ be a subgroup of $\operatorname{Aut}(\Gamma) . H$ is regular on $V(\Gamma)$ if

(1) $H$ is transitive on set $V(\Gamma)$,

(2) if $\sigma \in H$ satisfies that $\sigma(v)=v$ for any $v \in V(\Gamma)$, then $\sigma$ is the identity automorphism.

The following properties provides us a criterion to judge whether a graph is a Cayley graph, and we give a complete proof here for the convenience of readers.

Proposition 4. (([21], Lemma 16.3) ([16], Proposition 1.1)) A graph $\Gamma$ is a Cayley graph of a group $G$ if and only if $\operatorname{Aut}(\Gamma)$ contains a subgroup isomorphic to $G$ that is regular on $V(\Gamma)$.

Proof of Proposition 4. Supposed that $\Gamma$ is a Cayley graph $\operatorname{Cay}(G, S)$. For any $g \in G$, we define the assignment

$$
\bar{g}: u \mapsto u g^{-1}, \forall u \in V(\Gamma) .
$$

It is clear that $\bar{g}$ is a is a permutation on $V(\Gamma)$. Note that $v g^{-1}\left(u g^{-1}\right)^{-1} \in S$ if and only if $v u^{-1} \in S$ and thus $\bar{g} \in \operatorname{Aut}(\Gamma)$. Let $\bar{G}=\{\bar{g} \mid g \in G\}$, then $\bar{G} \leq \operatorname{Aut}(\Gamma)$ and $\bar{G} \cong G$. For any two vertices $u, v \in V(\Gamma)$, there exists a unique element $\bar{g}=\bar{v}^{-1} \bar{u} \in \bar{G}$ maps $u$ to $v$, and thus $\bar{G}$ acts transitively on $V(\Gamma)$. The uniqueness of $\bar{g}$ implies that $\bar{G}$ is a regular subgroup of $\operatorname{Aut}(\Gamma)$.

Conversely, we assume that $G$ is a subgroup of $\operatorname{Aut}(\Gamma)$ and regular on $V(\Gamma)$. We will show that $\Gamma$ is a Cayley graph of $G$. Let $V(\Gamma)=\left\{v_{i} \mid i=1, \ldots, n\right\}$, then $G$ has a unique element $g_{i}$ such that $g_{i}\left(v_{1}\right)=v_{i}$ since $G$ is regular. Set

$$
S=\left\{g_{j} \in G \mid v_{1} \text { is adjacent to } v_{j}\right\} .
$$

Now we define a map $f: \Gamma \rightarrow \operatorname{Cay}(G, S)$ sending $v_{i}$ to $g_{i}$. Then $v_{j}$ is adjacent to $v_{k}$ in $\Gamma$ if and only if $g_{j}\left(v_{1}\right)$ is adjacent to $g_{k}\left(v_{1}\right)$, if and only if $v_{1}$ is adjacent to $g_{j}^{-1} g_{k}\left(v_{1}\right)$, if and only if $g_{j}^{-1} g_{k} \in S$, and, finally, if and only if $g_{j}=f\left(v_{j}\right)$ is adjacent to $g_{k}=f\left(v_{k}\right)$ in Cay $(G, S)$. So $f$ is an isomorphism.

Let $\mathbb{F}_{q}$ be a finite field and $q$ be the power of odd prime numbers. By Definition 4 of uniform isomorphism in Section 2, it is straightforward to check that $B \Gamma_{2}\left(\mathbb{F}_{q} ; p_{1}^{2} l_{1}^{2}\right)$ without uniform automorphism (except identity automorphism) but $\phi$ is an automorphism.

Lemma 1. Let $\mathbb{F}_{q}$ be a finite field and $q$ be the power of odd prime numbers. Then $B \Gamma_{2}\left(\mathbb{F}_{q} ; p_{1}^{2} l_{1}^{2}\right)$ is not a Cayley graph.

Proof of Lemma 1. For any $x \in \mathbb{F}_{q}$, we consider the 3-path in $B \Gamma_{2}\left(\mathbb{F}_{q} ; p_{1}^{2} l_{1}^{2}\right):(1,0) \sim$ $\left[x, x^{2}\right] \sim(-1,0)$. There are at least $q$ paths between two points $(1,0)$ and $(-1,0)$. Suppose $B \Gamma_{2}\left(\mathbb{F}_{q} ; p_{1}^{2} l_{1}^{2}\right)$ is a Cayley graph, then it is a vertex transitive graph by Proposition 4 . Then there is $\sigma \in \operatorname{Aut}\left(B \Gamma_{2}\left(\mathbb{F}_{q} ; p_{1}^{2} l_{1}^{2}\right)\right)$ such that $\sigma((1,0))=(0,0)$. We get 3-path: $(0,0) \sim[y, 0] \sim$ $\left(p_{1}, p_{2}\right)$, where $\left(p_{1}, p_{2}\right)=\sigma((-1,0))$ and $p_{1} \neq 0$. Then $y^{2}=\frac{p_{2}}{p_{1}^{2}}$ and the number of 3-paths between $(0,0)$ and $\left(p_{1}, p_{2}\right)$ is strictly less than $q$, which contradicts that $\sigma$ is an automorphism.

We recall the definition of group of dihedral type.

Definition 8. ([15], Definition 2.21) A group $G$ is of dihedral type if it has an abelian subgroup $A$ of index 2, and an element $\pi \in G \backslash A$ of order 2 , such that $\pi g \pi=g^{-1}$ for all $g \in A$.

In this case, we write $G=\langle\pi, A\rangle$. Note that if $A$ is the cyclic subgroup of order $n$, then $G$ is precisely the dihedral group $D_{2 n}$.

Lemma 2. Let $|R|=r$. If $\mathcal{F}_{n}=\left\{f_{i} \mid i=2, \ldots, n\right\}$ is Cayley graphic of dihedral type in $R$, then the automorphism group of $B \Gamma_{n}\left(R ; f_{2}, \ldots, f_{n}\right)$ admits a subgroup is of dihedral type with $2 r^{n}$ elements. 
Proof of Lemma 2. For the graph $B \Gamma_{n}$, we have the automorphism $\phi:(p) \leftrightarrow[p]$, and the following automorphisms

$$
\begin{gathered}
\sigma_{k, x}: B \Gamma_{n} \longrightarrow B \Gamma_{n} \\
\left(p_{1}, \ldots, p_{k}, \ldots, p_{n}\right) \mapsto\left(p_{1}, \ldots, p_{k}+x, p_{k+1}+b_{k+1, k}, \ldots, p_{n}+b_{n, k}\right), \\
{\left[l_{1}, \ldots, l_{k}, \ldots, l_{n}\right] \mapsto\left[l_{1}, \ldots, l_{k}-x, l_{k+1}+y_{k+1, k}, \ldots, l_{n}+y_{n, k}\right],}
\end{gathered}
$$

where $x \in R$ and $k=1, \ldots, n$.

Next, we will prove that $A=\left\langle\sigma_{k, x}\right\rangle$ is an abelian group. We first show the commutativity of these generators, i.e., $\sigma_{k, x} \sigma_{k^{\prime}, z}=\sigma_{k^{\prime}, z} \sigma_{k, x}$ for any $k \leq k^{\prime}$ and $x, z \in R$.

Let $(p)=\left(p_{1}, \ldots, p_{n}\right)$ and $[l]=\left[l_{1}, \ldots, l_{n}\right]$. We denote $b_{k, k^{\prime}, i}+p_{i}, y_{k, k^{\prime}, i}+l_{i}, b_{k^{\prime}, k, i}+p_{i}$, $y_{k^{\prime}, k, i}+l_{i}$ for the $i$-th component of $\sigma_{k, x} \sigma_{k^{\prime}, z}((p)), \sigma_{k, x} \sigma_{k^{\prime}, z}([l]), \sigma_{k^{\prime}, z} \sigma_{k, x}\left((p), \sigma_{k^{\prime}, z} \sigma_{k, x}([l])\right.$ respectively, for $1 \leq i \leq n$.

It is straightforward to verify that $b_{k, k^{\prime}, i}=b_{k^{\prime}, k, i}$ and $y_{k, k^{\prime}, i}=y_{k^{\prime}, k, i}$ for $i \leq k^{\prime}$. For the case $k^{\prime}<i \leq n$, suppose that

$$
\begin{aligned}
F & =\left(p_{1}, l_{1}, \ldots, p_{k-1}, l_{k-1}, p_{k}+x, l_{k}-x\right), \\
G & =\left(p_{k+1}+b_{k+1, k}, l_{k+1}+y_{k+1, k}, \ldots, p_{k^{\prime}-1}+b_{k^{\prime}-1, k}, l_{k^{\prime}-1}+y_{k^{\prime}-1, k}\right), \\
H & =\left(p_{k^{\prime}+1}+b_{k, k^{\prime}, k^{\prime}+1}, l_{k^{\prime}+1}+y_{k, k^{\prime}, k^{\prime}+1}, \ldots, p_{i-1}+b_{k, k^{\prime}, i-1}, l_{i-1}+y_{k, k^{\prime}, i-1}\right), \\
I & =\left(p_{k^{\prime}}+b_{k^{\prime}, k}, l_{k^{\prime}}+y_{k^{\prime}, k}, p_{k^{\prime}+1}+b_{k^{\prime}+1, k}, l_{k^{\prime}+1}+y_{k^{\prime}+1, k} \ldots, p_{i-1}+b_{i-1, k}, l_{i-1}+y_{i-1, k}\right) .
\end{aligned}
$$

By induction on $i$, we have

$$
\begin{aligned}
& b_{k^{\prime}, k, i}-b_{i, k}+y_{k^{\prime}, k, i}-y_{i, k}=b_{k, k^{\prime}, i}-b_{i, k}+y_{k, k^{\prime}, i}-y_{i, k}, \\
& =f_{i}\left(F, G, p_{k^{\prime}}+b_{k^{\prime}, k}+z, l_{k^{\prime}}+y_{k^{\prime}, k}-z, H\right)-f_{i}(F, G, I) .
\end{aligned}
$$

Let the sum of all odd functions in the additive terms of $b_{k^{\prime}, k, i}$ be $h(x, z)(h(x, z)=$ $-h(-x,-z))$. If $h(x, z) \neq 0$, by Definition 5 and Proposition 2 , we have $\sigma_{k, x}((p))_{j}=$ $\sigma_{k,-x}([p])_{j}$ for all $1 \leq j \leq n$. Moreover, set $p_{m}=l_{m}$ for all $1 \leq m \leq n$, and

$$
\begin{aligned}
O_{b}\left(p_{1}, \ldots, p_{k}+x, p_{k+1}+b_{k+1, k}, \ldots, p_{i-1}+b_{i-1, k}, z\right) & =b_{k^{\prime}, k, i}-b_{i, k}-h(x, z), \\
O_{y}\left(l_{1}, \ldots, l_{k}-x, l_{k+1}+y_{k+1, k}, \ldots, l_{i-1}+y_{i-1, k}, z\right) & =y_{k^{\prime}, k, i}-y_{i, k}+h(x, z) .
\end{aligned}
$$
where

We have $O_{b}+O_{y}=b_{k^{\prime}, k, i}-b_{i, k}+y_{k^{\prime}, k, i}-y_{i, k}$, which contradicts Proposition 3 (1)

$$
(F, G, I)_{2 j-1}=(F, G, I)_{2 j} \text { for all } j=1, \ldots, i-1 .
$$

We have $h(x, z)=0$. Similarly, let the sum of all odd functions in the additive terms of $b_{k, k^{\prime}, i}$ be $h^{\prime}(x, z)$, then $h^{\prime}(x, z)=0$. With a similar discussion as in the proof of Proposition 3 , we have $\sigma_{k, x} \sigma_{k^{\prime}, z}=\sigma_{k^{\prime}, z} \sigma_{k, x}$ for any $x, z \in R$.

By Proposition $3(1)(2)$, we have $\sigma_{k, 0}=\mathrm{id}$ and $\sigma_{k, x}^{-1}=\sigma_{k,-x}$. By a straightforward check, we have $\phi \sigma_{k, x} \phi=\sigma_{k,-x}$, and $A=\left\langle\sigma_{k, x}\right\rangle$ is an abelian group, and thus $G=\langle\phi, A\rangle$ is of dihedral type. Moreover, it is clear that $|\phi|=2$, and for any $1 \leq k \leq n,\left\{\sigma_{k, x} \mid x \in R\right\}$ has $r$ elements. By the commutativity and $\sigma_{k, x} \sigma_{k, y}=\sigma_{k, x+y}$, the abelian group $A$ has $r^{n}$ automorphisms and thus $G=\langle\phi, A\rangle$ is a subgroup of $\operatorname{Aut}\left(B \Gamma_{n}\right)$ is of dihedral type with $2 r^{n}$ elements.

Now we are ready to prove our main theorem.

Theorem 4. The function system $\mathcal{F}_{n}=\left\{f_{i} \mid i=2, \ldots, n\right\}$ is Cayley graphic of dihedral type in $R$ if and only if $B \Gamma_{n}$ is a Cayley graph $\operatorname{Cay}(G, S)$ on some group $G=\langle\phi, A\rangle$ of dihedral type with $\phi:(p) \leftrightarrow[p]$ lies in $\operatorname{Aut}\left(B \Gamma_{n}\right)$ and $A$ uniform. 
Proof of Theorem 1. By Lemma 2 and Proposition 4, we can verify that $G$ is regular on $V\left(B \Gamma_{n}\right)$. We have $B \Gamma_{n}\left(R ; f_{2}, \ldots, f_{n}\right)$ is a Cayley graph $\operatorname{Cay}(G, S)$ where $G=\langle\phi, A\rangle$ is of dihedral type and $A$ is uniform.

If $B \Gamma_{n}$ is a Cayley graph $\operatorname{Cay}(G, S)$, by Definition 4 , then there is $\sigma_{k, x} \in A$, such that

$$
\begin{gathered}
\sigma_{k, x}((0))_{k}=x \text { and } \sigma_{k, x}((0))_{j}=0 \text { for all } 1 \leq j<k \leq n, \\
\sigma_{k, x}((p))_{k}=p_{k}+x \text { and } \sigma_{k, x}((p))_{j}=p_{j} \text { for all }(p) \in P_{n} .
\end{gathered}
$$

Since $\phi \sigma_{k, x} \phi=\sigma_{k, x}^{-1}$, we have

$$
\sigma_{k, x}([p])_{k}=p_{k}-x \text { and } \sigma_{k, x}([p])_{j}=p_{j} \text { for all }[p] \in L_{n} .
$$

By Proposition 2 and 3 (1), we have $\sigma_{k, x}^{-1}=\sigma_{k,-x}$ and then the theorem follows.

By Lemma 1 , there is a family of non-Cayley graphs $B \Gamma_{2}\left(\mathbb{F}_{q} ; p_{1}^{2} l_{1}^{2}\right)$ with $q$ the power of odd prime number that have no uniform automorphism (except identity automorphism) but satisfy $\phi \in \operatorname{Aut}\left(B \Gamma_{2}\right)$; therefore the above conditions in Definition 5 are necessary. By the previous theorem, we have two corollaries as follows.

Corollary 1. The graph $B \Gamma_{n}\left(R ; f_{2}, \ldots, f_{n}\right)$ is a Cayley graph in the following cases

(1) $f_{i}=\sum_{s=1}^{i-1} a_{s}\left(p_{s}+l_{s}\right)^{b_{s}}+c$, where the constants $a_{s}, c \in R$ and integers $b_{s}$;

(2) $n=2$ and $f_{2}=c p_{1} l_{1}$ with constants $c, 2^{-1} \in R$, where $R$ is a commutative ring;

(3) $n=2$ and $f_{2}=c\left(p_{1} l_{1}\right)^{k p}$ with constant $c \in R$, where $R$ is a commutative ring of characteristic $p$ with $k p$ odd and $2^{-1} \in R$;

(4) $n=2$ and $f_{2}=a_{1} p_{1} l_{1}+b_{1}\left(p_{1}+l_{1}\right)^{c_{1}}+c$ with constants $a_{1}, b_{1}, c, 2^{-1} \in R$, where $R$ is a commutative ring and $c_{1}$ is an integer;

(5) $n=2$ and $f_{2}=a_{1}\left(p_{1} l_{1}\right)^{k p}+b_{1}\left(p_{1}+l_{1}\right)^{c_{1}}+c$ with constants $a_{1}, b_{1}, c, 2^{-1} \in R$, where $R$ is a commutative ring of characteristic $p$ with $k p$ odd and $c_{1}$ an integer;

(6) $\quad f_{n}=\sum_{s=1}^{n-1} b_{s}\left(p_{s} l_{s}\right)+\sum_{s=1}^{n-1} c_{s}\left(p_{s}+l_{s}\right)^{d_{s}}+\sum_{s=1}^{n-1} e_{s}\left(p_{s} l_{s}\right)^{m p}+c$, and $f_{i}=\sum_{s=1}^{i-1} a_{i, s}\left(p_{s}+l_{s}\right)^{b_{i, s}}+d_{i}$ for $i=2, \cdots, n-1$, where $R$ is a commutative ring of characteristic $p$ with $m p$ odd, the constants $a_{i, s}, b_{s}, c_{s}, e_{s}, c, d_{i}, 2^{-1} \in R$, and $b_{i, s}, d_{s}$ integers.

Proof of Corollary 1. (1) Take $b_{i, k}=0, y_{i, k}=0$ and the statement follows.

(2) Define

$$
b_{2,1}=c\left(-p_{1} x-\frac{1}{2} x^{2}\right), y_{2,1}=c\left(l_{1} x-\frac{1}{2} x^{2}\right) .
$$

Then $\mathcal{F}_{2}$ is Cayley graphic of dihedral type, and $B \Gamma_{2}$ is a Cayley graph

(3) Define

$$
b_{2,1}=c\left(-\left(p_{1} x\right)^{k p}-\frac{1}{2} x^{2 k p}\right), y_{2,1}=c\left(\left(l_{1} x\right)^{k p}-\frac{1}{2} x^{2 k p}\right) .
$$

Then $\mathcal{F}_{2}$ is Cayley graphic of dihedral type, and $B \Gamma_{2}$ is a Cayley graph.

(4) and (5) follow by (1), (2), (3), and Proposition 3 (3).

(6) Set

$$
\begin{aligned}
& b_{n, k}=\sum_{s=1}^{n-1} b_{s}\left(-p_{s} x-\frac{1}{2} x^{2}\right)+\sum_{s=1}^{n-1} e_{s}\left(-\left(p_{s} x\right)^{m p}-\frac{1}{2} x^{2 m p}\right), \\
& y_{n, k}=\sum_{s=1}^{n-1} b_{s}\left(l_{s} x-\frac{1}{2} x^{2}\right)+\sum_{s=1}^{n-1} e_{s}\left(\left(l_{s} x\right)^{m p}-\frac{1}{2} x^{2 m p}\right),
\end{aligned}
$$


and $b_{i, k}=y_{i, k}=0$ for $k<i<n$. Then $B \Gamma_{n}$ is a Cayley graph.

Corollary 2. Let $R$ be a finite ring of characteristic 2 and $\mathcal{F}_{n}=\left\{f_{i} \mid i=2, \ldots, n\right\}$ be a Cayley graphic function system of dihedral type in $R$. If $\Gamma_{n}$ ha a loop at each vertex or no-loop at all vertices, then $\Gamma_{n}$ is a Cayley graph $\operatorname{Cay}(A, S)$ with $A$ an abelian group.

Proof of Corollary 2. By Definition 5 and Proposition 2, $b_{i, k}=y_{i, k}$ associated to $\mathcal{F}_{n}=\left\{f_{i} \mid i=2, \ldots, n\right\}$ is Cayley graphic of dihedral type in $R$.

For any $x \in R$ and $k=1, \ldots, n$, we define

$$
\begin{aligned}
\sigma_{k, x}: \Gamma_{n} & \longrightarrow \Gamma_{n} \\
\left\langle a_{1}, \ldots, a_{k}, \ldots, a_{n}\right\rangle & \longmapsto\left\langle a_{1}, \ldots, a_{k}+x, a_{k+1}+b_{k+1, k}, \ldots, a_{n}+b_{n, k}\right\rangle .
\end{aligned}
$$

The proof is similar to Lemma 2 and Theorem 4.

We conclude this section with two concrete examples.

Example 3. Assume $n=2$ and the function system $f_{2}=p_{1}+l_{1}$, we set

$$
\begin{aligned}
\phi: & \left(p_{1}, p_{2}\right) \mapsto\left[p_{1}, p_{2}\right] \\
{\left[l_{1}, l_{2}\right] } & \mapsto\left(l_{1}, l_{2}\right) \\
\sigma_{2, x}:\left(p_{1}, p_{2}\right) & \mapsto\left(p_{1}, p_{2}+x\right) \\
{\left[l_{1}, l_{2}\right] } & \mapsto\left[l_{1}, l_{2}-x\right] \\
\sigma_{1, x}:\left(p_{1}, p_{2}\right) & \mapsto\left(p_{1}+x, p_{2}\right) \\
{\left[l_{1}, l_{2}\right] } & \mapsto\left[l_{1}-x, l_{2}\right]
\end{aligned}
$$

Then for any $i, j=1$ or 2 and $x \in R$, we have

$$
\sigma_{i, x} \sigma_{j, y}=\sigma_{j, y} \sigma_{i, x}, \phi \sigma_{i, x} \phi=\sigma_{i,-x}
$$

Thus $B \Gamma_{2}\left(R ; p_{1}+l_{1}\right)$ is a Cayley graph.

If char $R=2$, by Proposition $1, \Gamma_{2}$ has loop at all vertices, we have $\Gamma_{2}\left(R ; p_{1}+l_{1}\right)$ is a Cayley graph.

Example 4. Let $n=2, f_{2}=p_{1} l_{1}$ and $R$ be a commutative ring with $2^{-1} \in R$, take

$$
\begin{aligned}
\phi: & \left(p_{1}, p_{2}\right) \mapsto\left[p_{1}, p_{2}\right] \\
{\left[l_{1}, l_{2}\right] } & \mapsto\left(l_{1}, l_{2}\right) \\
\sigma_{2, x}:\left(p_{1}, p_{2}\right) & \mapsto\left(p_{1}, p_{2}+x\right) \\
{\left[l_{1}, l_{2}\right] } & \mapsto\left[l_{1}, l_{2}-x\right] \\
\sigma_{1, x}:\left(p_{1}, p_{2}\right) & \mapsto\left(p_{1}+x, p_{2}-p_{1} x-\frac{1}{2} x^{2}\right) \\
{\left[l_{1}, l_{2}\right] } & \mapsto\left[l_{1}-x, l_{2}+l_{1} x-\frac{1}{2} x^{2}\right]
\end{aligned}
$$

For any $i, j=1$ or 2 and $x \in R$, we have

$$
\sigma_{i, x} \sigma_{j, y}=\sigma_{j, y} \sigma_{i, x}, \phi \sigma_{i, x} \phi=\sigma_{i,-x}
$$

Then $B \Gamma_{2}\left(R ; p_{1} l_{1}\right)$ is a Cayley graph.

\section{The Hamiltonicity of Graph $B \Gamma_{n}$}

Based on the previous section, this section is devoted to considering the Hamiltonicity of graph $B \Gamma_{n}$. 
We recall two definitions related to the Hamiltonicity of graphs.

Definition 9. ([22], Section 4.2) A path that contains every vertex of graph $\Gamma$ is called a Hamilton path of $\Gamma$; similarly, a Hamilton cycle of $\Gamma$ is a cycle that contains every vertex of $\Gamma$. A graph is a Hamiltonian graph or Hamiltonian if it contains a Hamilton cycle.

Definition 10. ([23,24]) A bipartite graph with parts $A$ and $B$ is Hamilton-laceable if, for all vertices $u \in A$ and $v \in B$, there is a Hamilton path whose terminal vertices are $u$ and $v$.

The following two lemmas, originally from $[17,25]$, study the group on which the Cayley graph is Hamiltonian.

Lemma 3. ([17], Corollary 2) If $G$ is a abelian group, then every connected Cayley graph on $G$ has a Hamilton cycle.

Lemma 4. ([25], Theorem 6.1) If G is p-group, then every connected Cayley graph on G has a Hamilton cycle.

As a generalization of Lemma 4, the proof of the following lemma can be reduced to Lemma 4.

Lemma 5. ([19], Corollary 3.3) Let $G$ be a finite group with $S$ a subset of $G$. If, moreover,

(1) $S$ is a generating set of $G$,

(2) $A$ is a normal $p$-subgroup of $G$, for some prime $p$, and

(3) $s t^{-1} \in A$, for all $s, t \in S$,

then $\operatorname{Cay}(G, S)$ has a Hamilton cycle.

The following lemma is useful to our discussion on the Hamiltion-laceablity of the graph.

Lemma 6. ([23], Theorem 1.7) Let $G$ be a finite group of dihedral type and Cayley graph $\Gamma$ on group $G$ be connected bipartite. If the order of $G$ is divisible by 4 and $\Gamma$ of valency at least 3 , then $\Gamma$ is Hamilton-laceable.

Based on our discussion in the previous section, we establish two Hamiltonian properties about graph $B \Gamma_{n}$ as follows.

Theorem 5. Let $R$ be a finite ring with $|R|$ an even positive integer, and $B \Gamma_{n}$ be a connected graph detemined by the function system $\mathcal{F}_{n}=\left\{f_{i} \mid i=2, \ldots, n\right\}$, which is Cayley graphic of dihedral type. Then $B \Gamma_{n}$ is Hamilton-laceable.

Proof of Theorem 2. If $r=|R|=2$, the graph $B \Gamma_{n}$ is a cycle since $B \Gamma_{n}$ is a 2-regular graph and connected. For other cases of $r$, by Lemma 2 and Theorem 4 , the graph $B \Gamma_{n}$ is Cayley graph on group $G$ of dihedral type with order $2 r^{n}$. Since $r=|R|>3$ is an even integer, the order of $G$ can be divided by 4 and the valency of $B \Gamma_{n}$ is at least 3 . Thus $B \Gamma_{n}$ is Hamilton-laceable by Lemma 6.

Theorem 6. Let $R=G F\left(p^{k}\right)$ be a field, for some prime $p$. If $\mathcal{F}_{n}=\left\{f_{i} \mid i=2, \ldots, n\right\}$ is Cayley graphic of dihedral type in $R$ and $B \Gamma_{n}$ is connected, then $B \Gamma_{n}$ has a Hamilton cycle.

Proof of Theorem 3. Note that the graph $B \Gamma_{n}$ is Cayley graph on group $G$ of dihedral type by Theorem 4 . To prove the theorem, it suffices to check the conditions in Lemma 5 one by one. 
(1) Note that $S$ is a generating set of $G$ if and only if $\operatorname{Cay}(G, S)$ is connected.

(2) By Lemma 2, we have $B \Gamma_{n}=\operatorname{Cay}(G, S), G=\langle\phi, A\rangle$ is of dihedral type, and $|A|=r^{n}=p^{k n}$. Hence, $A$ is a normal $p$-subgroup of $G$.

(3) By Proposition 4 and $B \Gamma_{n}=\operatorname{Cay}(G, S)$ is a bipartite graph. According to the structure of $S$ and $G$, we have the element in $S$ is $s=a \phi$ or $\phi a$, where $a \in A$. Hence, $s t^{-1} \in A$ for all $s, t \in S$.

A variant of the well-known Lovász Conjecture on Hamiltonian paths states that every finite connected Cayley graph has a Hamilton cycle. Note that both Theorem 6 and the following two corollaries support this conjecture.

The following example shows that $B \Gamma_{2}\left(G F(2) ; p_{1} l_{1}\right)$ is a Hamiltonian graph and Cayley graph $\operatorname{Cay}(A, S)$ where $A$ is an abelian group.

Example 5. Let $R=G F(2)$ be a field. Then $B \Gamma_{2}\left(R ; p_{1} l_{1}\right)$ is a Cayley graph and has a Hamilton cycle (see Figure 1).

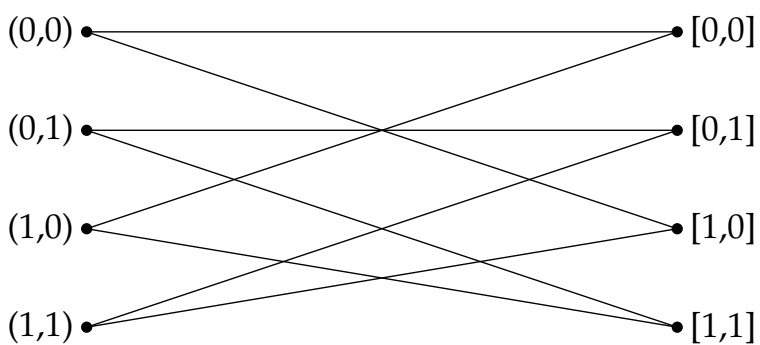

Figure 1. The graph $B \Gamma_{2}\left(R ; p_{1} l_{1}\right)$.

Note that $B \Gamma_{2}\left(\mathbb{Z}_{2} ; p_{1} l_{1}\right)$ is precisely a Cayley graph Cay $(A, S)$ with $A$ is a cyclic group generated by the permutation $(12345678) \in S_{8}$ and $S=\left\{(12345678),(12345678)^{-1}\right\}$.

By Theorem 2.1 (3) in [14], if $R=G F(q)$ is a finite field with $q$ a prime power, then $B \Gamma_{2}\left(R ; p_{1} l_{1}\right)$ is connected. The following corollary implies that graph $B \Gamma_{2}\left(R ; p_{1} l_{1}\right)$ is Hamiltonian where $q$ is an odd prime power, which supports the conjecture that Wenger graph has pancyclicity in [26].

Corollary 3. Let $p$ be an odd prime and $R=G F\left(p^{k}\right)$ be a finite field. Then graphs $B \Gamma_{2}\left(R ; p_{1} l_{1}\right)$ and $B \Gamma_{2}\left(G F(2) ; p_{1} l_{1}\right)$ have a Hamilton cycle.

Proof of Corollary 3. The graph $B \Gamma_{2}\left(G F(2) ; p_{1} l_{1}\right)$ has a Hamilton cycle by Figure 1 . If $p$ is an odd prime, by Corollary $1, B \Gamma_{2}\left(R ; p_{1} l_{1}\right)$ is a Cayley graph. To prove the connectivity, we need to find out the connection set $S$ of Cayley graph $B \Gamma_{2}\left(R ; p_{1} l_{1}\right) \cong \operatorname{Cay}(G, S)$. Indeed, we have $\phi \sigma_{2, \frac{x^{2}}{2}} \sigma_{1, x} \in S$ since $(0,0) \sim[x, 0]$ for all $x \in G F\left(p^{k}\right)$. Take $x=0$ and we have $\phi \in\langle S\rangle$. Therefore $\sigma_{2, x^{2}}$ and $\sigma_{2,-x^{2}}$ lie in $\langle S\rangle$, which implies $\sigma_{2,-\frac{x^{2}}{2}} \in\langle S\rangle$ and $\sigma_{1, x} \in\langle S\rangle$ for all $x \in G F\left(p^{k}\right)$. Since the equation $x^{2}+y^{2}=z$ has a solution $(x, y)$ for any $z$ in $G F\left(p^{k}\right)$, the automorphism $\sigma_{2, z}$ can be written as the composition of $\sigma_{2, x^{2}}$ and $\sigma_{2, y^{2}}$ in $\langle S\rangle$, thus $\sigma_{2, z} \in\langle S\rangle$, which implies $G=\langle S\rangle$, and then $B \Gamma_{2}\left(R ; p_{1} l_{1}\right)$ is connected, so $B \Gamma_{2}\left(R ; p_{1} l_{1}\right)$ has a Hamilton cycle by Theorem 6.

For the graph $\Gamma_{n}$, we have the following corollary.

Corollary 4. Let $R$ be a finite ring of characteristic 2 and $\Gamma_{n}$ be a connected graph. If $\mathcal{F}_{n}$ is Cayley graphic of dihedral type and $\Gamma_{n}$ has loop or no-loop at all vertices, then $\Gamma_{n}$ has a Hamilton cycle.

Proof of Corollary 4. It follows directly by Corollary 2 and Lemma 3. 


\section{Conclusions}

In Theorem 4 of this paper, under the conditions (see Definition 5) proposed in this paper, we obtain the necessary and sufficient condition that the graph $B \Gamma_{n}$ is Cayley graph on some group $G=\langle\phi, A\rangle$ of dihedral type with $\phi:(p) \leftrightarrow[p]$ lies in $\operatorname{Aut}\left(B \Gamma_{n}\right)$ and $A$ uniform. Moreover in this case, we discuss the Hamiltonicity of $B \Gamma_{n}$. However, it has not been completely described when $B \Gamma_{n}$ is a Cayley graph or Hamiltonian graph. It will be interesting to consider the pancyclicity of a Wenger graph for our future work (see [26]).

Author Contributions: Writing-original draft preparation, F.Y. and C.Z.; writing-review and editing, Q.S. and H.Z. All authors have read and agreed to the published version of the manuscript.

Funding: This work is supported by National Natural Science Foundation of China (Grant No.11971196, 11961007) and Science Technology Foundation of Guizhou Province (Grant No. [2020]1Y405).

Institutional Review Board Statement: Not applicable.

Informed Consent Statement: Not applicable.

Data Availability Statement: No data were reported in the study.

Acknowledgments: The authors thank Zhang Shunzhe for his advices on this paper. Moreover, the authors are grateful to the referees for many helpful comments.

Conflicts of Interest: The authors declare no conflict of interest.

\section{References}

1. Lazebnik, F.; Ustimenko, V.A. New examples of graphs without small cycles and of large size. Eur. J. Comb. 1993, 14, 445-460. [CrossRef]

2. Lazebnik, F.; Ustimenko, V.A.; Woldar, A.J. Polarities and 2k-cycle-free graphs. Discret. Math. 1999, 197, 503-513. [CrossRef]

3. Lazebnik, F.; Woldar, A.J. General properties of some families of graphs defined by systems of equations. J. Graph Theory 2001, 38, 65-86. [CrossRef]

4. Woldar, A.J. On generalizing generalized polygons. Innov. Incid. Geom. Algebr. Topol. Comb. 2010, 10, 147-170. [CrossRef]

5. Terlep, T.A.; Williford, J. Graphs from Generalized Kac-Moody Algebras. Siam J. Discret. Math. 2012, 26, 1112-1120. [CrossRef]

6. Lazebnik, F.; Mubayi, D. New lower bounds for Ramsey numbers of graphs and hypergraphs. Adv. Appl. Math. 2002, 28, 544-559. [CrossRef]

7. Lazebnik, F.; Ustimenko, V.A.; Woldar, A.J. New constructions of bipartite graphs on $m$, $n$ vertices with many edges and without small cycles. J. Comb. Theory Ser. B 1994, 61, 111-117. [CrossRef]

8. Lazebnik, F.; Ustimenko, V.A. Explicit construction of graphs with an arbitrary large girth and of large size. Discret. Appl. Math. 1995, 60, 275-284. [CrossRef]

9. Lazebnik, F.; Sun, S.; Wang, Y. Some families of graphs, hypergraphs and digraphs defined by systems of equations: A survey. Lect. Notes Semin. Interdiscip. Mat. 2017, 14, 105-142.

10. Cheng, X.; Chen, W.; Tang, Y. On the girth of the bipartite graph $D(k, q)$. Discret. Math. 2014, 335, 25-34. [CrossRef]

11. Cheng, X.; Chen, W.; Tang, Y. On the conjecture for the girth of the bipartite graph $D(k, q)$. Discret. Math. 2016, 339, 2384-2392. [CrossRef]

12. Ustimenko, V.A.; Woldar, A.J. Extremal properties of regular and affine generalized polygons as tactical configurations. Eur. J. Comb. 2003, 23, 99-111. [CrossRef]

13. Hou, X.; Lappano, S.D.; Lazebnik, F. Proof of a conjecture on monomial graphs. Finite Fields Their Appl. 2017, 43, 42-68. [CrossRef]

14. Lazebnik, F.; Viglione, R. An infinite series of regular edge-but not vertex-transitive graphs. J. Graph Theory 2002, 41, 249-258. [CrossRef]

15. Kutnar, K.; Marusic, D.; Morris, D.W.; Morris, J.; Sparl, P. Hamiltonian cycles in Cayley graphs whose order has few prime factors. ARS Math. Cont. 2012, 5, 27-71. [CrossRef]

16. Li, C.H. On isomorphisms of finite Cayley graphs-A survey. Discret. Math. 2002, 256, 301-334. [CrossRef]

17. Chen, C.C.; Quimpo, N.F. On strongly hamiltonian abelian group graphs. In Combinatorial Mathematics VIII; Springer: Berlin/Heidelberg, Germany, 1981; pp. 23-34.

18. Curran, S.J.; Gallian, J.A. Hamiltonian cycles and paths in Cayley graphs and digraphs-a survey. Discret. Math. 1996, 156, 1-18. [CrossRef]

19. Morris, D.W. 2-generated cayley digraphs on nilpotent groups have hamiltonian paths. Contri. Discret. Math. 2012, 7, 41-47.

20. Montellano-Ballesteros, J.J.; Arguello, A.S. Hamiltonian normal Cayley graphs. Discuss. Math. Graph Theory $2019,39,731-740$.

21. Biggs, N. Algebraic Graph Theory; Cambridge University Press: New York, NY, USA, 1992.

22. Bondy, J.A.; Murty, U.S.R. Graph Theory with Applications; Macmillan: London, UK, 1976. 
23. Alspach, B.; Chen, C.C.; Dean, M. Hamilton paths in Cayley graphs on generalized dihedral groups. ARS Math. Cont. 2010, 3, 29-47. [CrossRef]

24. Hsieh, S.Y.; Kuo, C.N. Hamiltonian-connectivity and strongly Hamiltonian-laceability of folded hypercubes. Comput. Math. Appl. 2007, 53, 1040-1044. [CrossRef]

25. Witte, D. Cayley digraphs of prime-power order are hamiltonian. J. Comb. Theory Ser. B 1986, 40, 107-112. [CrossRef]

26. Wang, Y.; Lazebnik, F.; Thomason, A. On some cycles in Wenger Graphs. Acta Math. Appl. Sin. Engl. Ser. 2020, 36, 492-502. [CrossRef] 